\title{
Application of a Novel Stability Control System for Coordination of Power Flow Control Devices in the Future GB Transmission System
}

\author{
S. K. Kerahroudi, Graduate Student Member, IEEE. \\ G. A. Taylor, Senior Member, IEEE. \\ Brunel Institute of Power Systems (BIPS) \\ Brunel University \\ London, UK.
}

\begin{abstract}
Following the increase in development of large-scale renewable energy sources in the UK and the pressing need for adequate transmission capacity to accommodate the upcoming renewable generation, more state-of-the-art power flow control devices such as embedded High Voltage DC (HVDC) links will come into operation in the future GB transmission system to provide the additional capacity. However, an operational supervisory stability control system is required to ensure the coordinated control of power flow control devices in order to achieve optimal dynamic performance and make stability limits a less binding factor than thermal limits. The focus of this paper is to demonstrate the capability of the multi variable controller for coordinated control using a non-parametric sampled regulator control design method. This method is very attractive for applications in large power systems since the complexity of the controller design would not increase by size of the power system. Also, in this study, this design method has been demonstrated for two power system applications.
\end{abstract}

Index Terms-Sampled Regulator, Stability Control and HVDC link.

\section{INTRODUCTION}

In line with UK and European $\mathrm{CO} 2$ reduction legislation the UK generations mix is changing to incorporate an increasing volume of renewable energy predominantly in the form of offshore wind generation [1], [2]. Thus, it is predicted that the installed capacity of wind generation will increase to around $30 \mathrm{GW}$ by 2020 compared to the current installed capacity of $5.5 \mathrm{GW}$ that include $2 \mathrm{GW}$ offshore [3]. The majority of the new wind generation will be in Scotland and offshore in the UK as a whole. Such an increase in variable renewable generation will result in a significant increase in the required power transfer between Scottish Power Transmission (SPT) and National Grid (NG) networks (known as the Anglo-Scottish boundary) [3]. The Anglo-Scottish boundary contains two double $400 \mathrm{kV}$ AC lines, with the current boundary capacity being stability limited to $2.5 \mathrm{GW}$. The stable operation of the network following a certain disturbance is guaranteed by reducing the power transfer on this boundary.

This research was supported by the Engineering and Physical Sciences Research Council (EPSRC) and National Grid plc, UK.

\author{
Fan. Li, Martin E. Bradley \\ Market Operation, \\ National Grid \\ Wokingham, UK
}

In addition, the ranges of the required power transfer capability increases every year due to the increase in the potential output from the intermittent wind generations [3]. As a result it is essential that the GB system operator and owners maximise the use of existing transmission lines and improve the stability limit in order to operate the transmission lines closer to their thermal limits and avoid constraining some generation plant. Therefore, NG have put a huge investment into this area: i.e. conductor upgrades have been ongoing in the last few years; new transmission technologies such as Thyristor Controlled Series Capacitor (TCSC) and embedded High Voltage DC (HVDC) links down the East and West coast are coming into operation in the GB system by 2016 and 2018 respectively. Hence, the network in this area is now congested with many types of new and automatic control devices. There is therefore, a risk of negative interaction between all control devices i.e. interaction between Power Oscillation Damping (POD) from Shunt Voltage Control (SVC) and HVDC link, interaction between devices with the flow control such as West coast HVDC link fighting against the regulation from TCSC or future East coast HVDC link. Ultimately, these power flow control devices could potentially affect the system stability and without investment in supervisory stability control systems much of the potential advantages and effectiveness of all these costly reinforcements on the transmission system could be wasted. Consequently, this will result in constraining the output of renewable energy sources which ultimately increases the operating costs of the UK electricity system. Therefore, a stability control system is required to guarantee the optimal dynamic performance and co-ordination of power flow control at pre-fault and enhance the stability limit at post-fault in such a large integrated power system.

\section{FEEDBACK CONTROL SYSTEM DESIGN}

In a control system as well as this study the SVC and HVDC link are regarded as actuators. The controller term used here refers to the devices that produce the control command signals based on various system measurements. 


\section{A. Primary and secondary control system}

Internal control systems within the actuators (in case of HVDC link and SVC) are primarily required to perform rapid pre- and post-fault voltage or flow regulations control. The secondary control is often used for servo-control systems where the primary control can regulate the controlled variables around command points. Although, both options (primary and secondary control) can be developed into either Single InputSingle Output (SISO) or Multi-Input and Multi-Output (MIMO) control systems, the internal controllers from manufacturers are usually based on the SISO design and tuned only for a single control variable. SISO design may be sufficient for the cases where the individual controls are near 'optimal' on their own and variables under control in the system are relatively uncoupled. The built-in SISO controllers will not be able to coordinate several individual actuators and control all outputs coherently in the feedback control system. With the presence of interactions the overall system stability and control performance can be severely compromised. Similar to linearity, interactions in a real system may depend on the system operating conditions. Within power systems, planned or unplanned outages may change the interactions between control variables and sometimes this can be fairly rapid and severe. In these cases control systems designed based on the SISO control will be challenged.

\section{MUlTI-VARIABLE SAMPLED REGULATOR}

This section will introduce the fundamental technique of the Sample Regulator (SR) control system design method. The non-parametric approach applied to the power flow coordination problem is a sampled Multi-variable control law which is based on the principle of a passivity energy system to ensure the closed loop system stability [4]-[7]. The SR controller algorithm which is explained below is particularly formulated for computer implementation.

\section{A. Algorithm}

Consider a MIMO stable linear time invariant plant $\mathrm{P}$ with unit step response $\mathrm{H}(\mathrm{t})$ and with the sampling rate of $\mathrm{T}$. The sampled output of the system is given by the output vector $\mathrm{y}(\mathrm{nT})$ as described in [5], [6].

$$
\begin{aligned}
& y(n T)=\sum_{r=1}^{n} \Delta H(r T) U((n-r) T) \\
& \Delta H(r T)=H(r T)-H((r-1) T) \quad n=0,1,2, \ldots
\end{aligned}
$$

Where the input vector $\mathrm{U}(\mathrm{t})$ is defined as:

$$
U(n T)= \begin{cases}0 & t<0 \\ U(n T) & t \in[n T,(n+1) T] \quad n=0,1,2 .\end{cases}
$$

Authors of the study presented in [6] developed a new method ensuring system stability by designing the closed loop system to be passive for the class of the sampled regulators of the form:

$$
U(n T)=c\left(y^{0}(n T)-y(n T)\right)+\sum_{r=0}^{n} b_{n-r} U_{r}
$$

In this MIMO system $\mathrm{U}$ and $\mathrm{y}$ are vectors and $\mathrm{C}$ and $\mathrm{b}$, with compatible dimensions, are constant matrixes that are calculated as follows:

$$
\begin{gathered}
C=\left(I+\sum_{n=1}^{\infty} \varphi_{n}\right) H(\infty T)^{-1} \\
b_{n}=C \Delta H(n T)-\varphi_{n} \quad b_{0}=0 \quad n>0
\end{gathered}
$$

It is proven in [6] that if the sequence of $\varphi n$ is selected to be passive the system with the sampled control law of (4) is asymptotically stable with zero Steady-State Error (SSE) with a quantifiable degree of robustness in the case of variations in the step response $H(t)$. Passivity of sequence $\{\varphi n\}$ is guaranteed if $\{\varphi n\}$ is real symmetric positive semi-definite, such that $\Sigma \varphi n$ converges and $(\varphi n-2 \varphi n+1+\varphi n+2)$ is also positive, semi-definite, for $\mathrm{n} \geq 0$. One simple choice for such a passive sequence is a sequence of real positive semi-definite matrix $\{\mathrm{I}, \mathrm{A}, \mathrm{A} 2, \mathrm{~A} 3, \ldots \mathrm{An} \ldots\}$ with $0<\mathrm{A}<\mathrm{I}$. With selecting $\varphi n=A n$, where A satisfies passivity conditions, being a symmetric positive semi-definite matrix, there is still a degree of freedom in the choice of A. It is proven in [6] that summed squares of system tracking error, which are shown in (7) is minimized by choosing A=I-B-1.

$$
\sum_{n=1}^{\infty}\left|y(n T)_{r e f}-y(n T)\right|^{2}
$$

Matrix B is the solution of the Lyapnov equation in (8):

$$
B \Gamma K+K Г B=-\left(K \Omega+\Omega^{T} K\right)
$$

Where:

$$
\begin{gathered}
\Gamma=\sum_{n=1}^{\infty}\left(\Delta H_{n}\right)^{T}\left(\Delta H_{n}\right) \\
K=H(\infty)^{T} H(\infty) \\
\Omega=\sum_{n=1}^{\infty}\left(\Delta H_{n}\right)^{T}\left(H_{n-1}-H(\infty)\right)
\end{gathered}
$$

This choice of A ensures both the stability and the optimal dynamic performance and hence guarantees the system damping and the tracking speed. This approach is a multivariable system design method. It follows feedback control laws and is a natural extension of the traditional (sampled) integrating regulator. In fact the integrator controller is a special case in this class of $S R$, when $b 1=1$ and $b n=0$ for $n>1$ in (4):

$$
U_{n}=C\left(y_{r e f}(n T)-y(n T)\right)+b_{1} U_{n-1}
$$




\section{B. Main features of the SR control.}

Since all information required in the design is obtained in the time domain, this algorithm is suitable for implementation of a computer controlled system. This algorithm could be further developed into an adaptive self-tuning on-line control algorithm. The self-tuning implementation of this regulator is to address changes in operating conditions and system configurations. It is noted that although the requirement on an open loop system time response implies the open-loop stability of the system. This does not limit this class of regulators to be useful to the power system control, since the intact power systems should be stable at a physically feasible operating point even without the flow control. Also, the stability of the design is independent of the sampling rate. However, control can be realistically implemented by a proper choice of the sampling rate in order to restrain the control signal to be within the physical limits of actuating devices.

\section{DEVELOPMENT OF MIMO SR CONTROLLER}

\section{A. Fundamental of HVDC link's technology}

The principle of HVDC technologies is explained in detail in [10]. The West and East coast HDVC links in the GBSO network is due to be in by 2016 and 2018 respectively. The Eastern HVDC link's technology is not decided yet. However, the type of HVDC technology wouldn't affect the secondary controller design. The Western link HVDC is planned to be a bi-pole Current Source Control (CSC)-HVDC design with maximum capacity of $2.2 \mathrm{GW}$. Also, the West HVDC converter is assumed to feature a short-term overload capability of nearly $10 \%$ up to $2.4 \mathrm{GW}$ with fast ramp-up capability within $400 \mathrm{~ms}$.

\section{B. Structure of secondary control for HVDC links}

The overall structure and framework of the MIMO SR controller for the HVDC links, first study case, is depicted schematically in Fig.1 (The structure of MIMO SR controller for the second study case is also similar to this case). The PowerFactory (PF) DIgSILENT to Matlab interface capability has been used to expand the capability of PF for the design of MIMO SR controller [8], [9]. The Matlab SR control slot shown in Fig.1 represents the externally designed SR controller which provides calculated control signals (U1and U2) to links to track the new set-point. The PF model represents the power system. The control of the bi-pole setpoint can be set under a single command point.

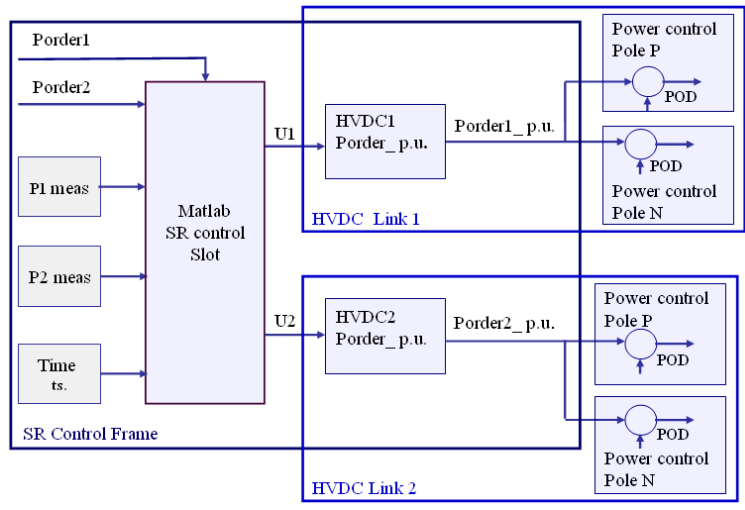

Figure 1. Implementation of MIMO SR control for the HVDC links.

\section{CONCEPT PROVING TEST SYSTEMS}

The SR controller design has been demonstrated for two power system applications: one for coordination of HVDC links and one for coordination of HVDC and SVC control.

\section{A. Coordinated control of HVDC links (study case1)}

A Single Machine-Infinite Bus (SMIB) test system, shown in Fig.2, is set up in PowerFactory to represent the transmission network connections between the North of England and Scotland. In this test system $2 \mathrm{GW}$ of power is transferred from Generator 1 to load and each HVDC link carries 500MW of power. Also, a dynamic model of the generator and CSC-HVDC links are implemented.

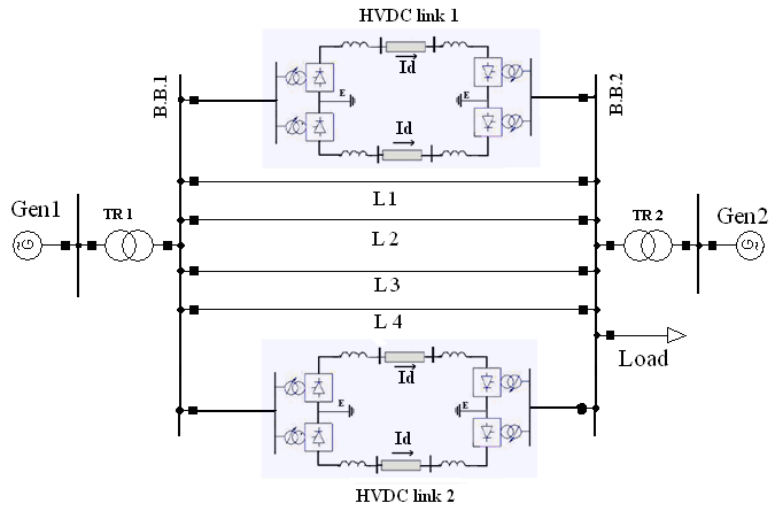

Figure 2. Concept proving PF test model for study case1.

For the first study case the performance of MIMO SR controller is investigated by conducting the following tests:

1) Pre-fault control: when a step change for one of the HVDC link is applied at pre-fault.

2) Post-fault control: when a $100 \mathrm{~ms}$, three phase fault occurred on one of the AC lines followed by fault clearance and post-fault action of both links after $400 \mathrm{~ms}$.

The first test allows investigation in to capability of MIMO SR controller for pre-fault coordinated control of power flow devices such as HVDC link as well as exploring the impact of control by varying the SR control parameters such as the sampling rate (T). Whereas, the second test examines the ability of the SR controller design for a robust control following a short circuit fault. The integration step size for all the simulations is $10 \mathrm{~ms}$ and the open loop time responses are truncated at $30 \mathrm{~s}$ of the simulation.

\section{B. Coordinated Control of HVDC link, SVC (study case 2)}

In the second test, a simple test model with initial transfer level of $2 \mathrm{GW}$ and 500MW power on HVDC link (shown in Fig.3) is set up for the control of the HVDC and SVC together under a single MIMO SR feedback control. In this case, the controller would address two very different control variables, one is power flow and the other is voltage, while the interactions are coupled through the rest of the transmission system. The control objectives are: the SVC control regulates the voltage profile by varying the shunt susceptance while the HVDC control tracks the wide range of operating point changes, at both pre-fault and post-fault. In this study case the 
performance of the MIMO SR controller for coordinated control of HVDC link and SVC is investigated only at prefault by applying a change of set point (Porder) by $100 \mathrm{MW}$.

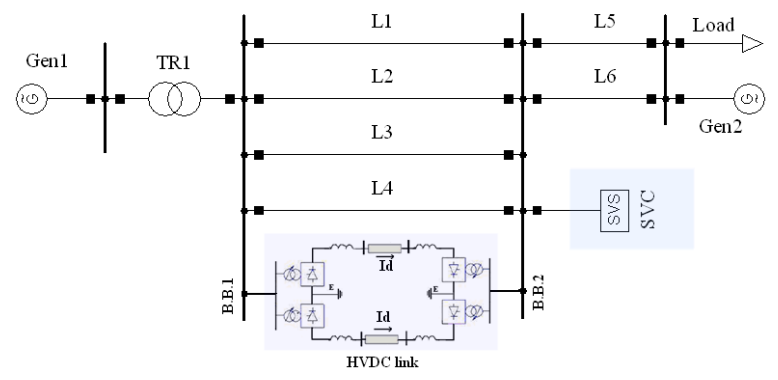

Figure 3. Concept proving PF test model for study case2.

\section{SIMULATION RESULTS AND ANALYSIS}

\section{A. Simulation results of study case1}

The simulation results of the first study case, presented in Fig.4, show the time responses of the HVDC links control system when a step change is imposed on the first HVDC link set-point with the second HVDC remaining at the original setpoint. Fig.4.a and Fig.4.b show the active power flows on the first and second HVDC link respectively after the change of HVDC link1 set-point. The red lines are the set-point of HVDC links and the green curves are the HVDC flow under the control of the internal SISO PI controller (with no cascade control). Also, the dark blue and light blue curves represent the MIMO SR controller performance with a $80 \mathrm{~ms}$ and $10 \mathrm{~ms}$ sampling rate correspondingly. The control signals (with various sampling time) generated from SR controller are shown in Fig.4.c. Clearly, it is evident from Fig.4.b (dark blue line) that following the change of set-point of one HVDC link, the interaction and impact on the other link are reduced significantly using the MIMO SR control with 80ms sampling
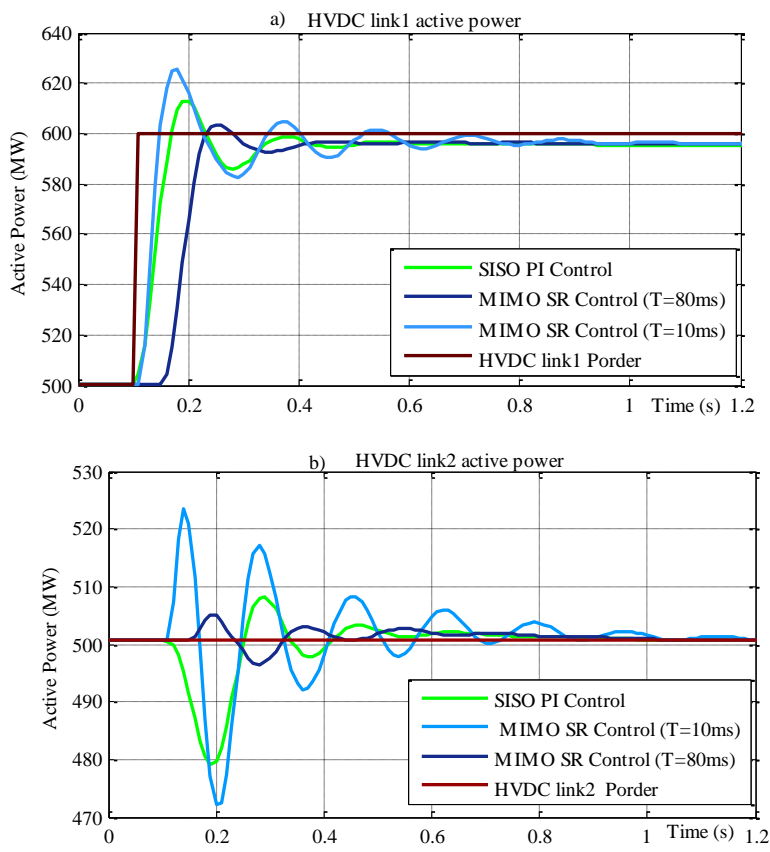

rate, whereas with having individual SISO PI controllers for each HVDC link, interaction is higher and can't be controlled.

In addition, the result of the post-fault control action test is presented in Fig.4.d. The system is at the operating point where $2 \mathrm{GW}$ is transferred from Gen1 to the load side. A $100 \mathrm{~ms} 3$-Phase short circuit fault is applied to one of the AC lines. The HVDC link has managed the 'blocking' mode and the voltage recovered soon after the fault has cleared. The setpoints of both HVDC links are changed from the original $500 \mathrm{MW}$ to $800 \mathrm{MW}$ at this point to compensate the loss of the faulted AC line and maintaining system synchronism. However, if the HVDC commutation failure occurs the control of the HVDC over the fault period can significantly deteriorate. Therefore, it is critical for stability control that the post-fault action from the HVDC link is avoided and instead special protection actions (generation inter-trip) are armed to cover the risk of such a failure of the feedback control system. It is important to notice that in the power flow control, the HVDC link is primarily to control power flow and enhance the boundary power transfer capacity while at the immediate postfault, the HVDC link fast ramping capacity and short term overload capabilities (up to $10 \%$ of total capacity) can be used to enhance the transient stability. Therefore, the main objective of the controller at post-fault is maintaining the system stability and that has more priority compared to postfault coordinated operation. Although, during the fault ridethrough period with the loss of a circuit the system configuration has been significantly changed and system has been subjected to various stages of non-linear evolutions, the SR control design based on the linear time responses around the pre-fault operation point has managed to robustly control the post-fault period and this system change has been tolerated by the robustness of the SR design and as can be seen from Fig.4.d, post fault performance of SR controller is as robust as SISO PI controller.
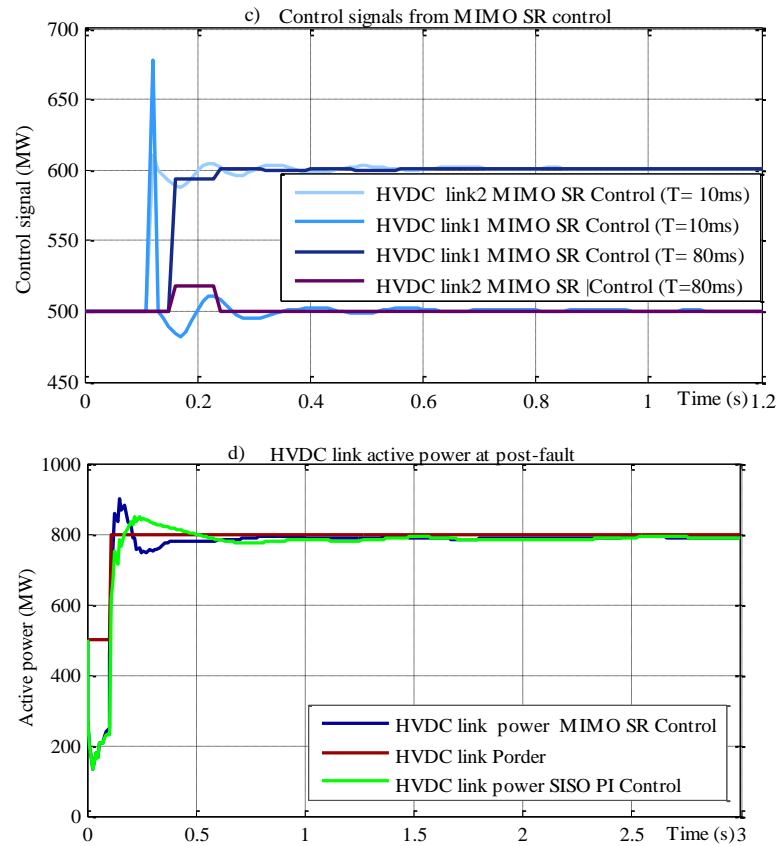

Figure 4. Comparison of SISO PI and MIMO SR controller performance for coordinated control of HVDC links. 


\section{B. Simulation results of study case 2}

Furthermore, the performance of MIMO SR controller for coordinated control of HVDC and SVC under a single frame work is investigated. The responses of the system when the HVDC link set-point is increased by $100 \mathrm{MW}$ at pre-fault are shown in Fig.5. It is seen from the Fig.5.b, green curve, that under normal operation condition, the step change in the HVDC power flow control (Fig.5.a) causes significant disturbance in the SVC voltage control when the system is under the SISO PI control. Whereas the interaction on voltage control is reduced using a MIMO SR control with $80 \mathrm{~ms}$ sampling rate.
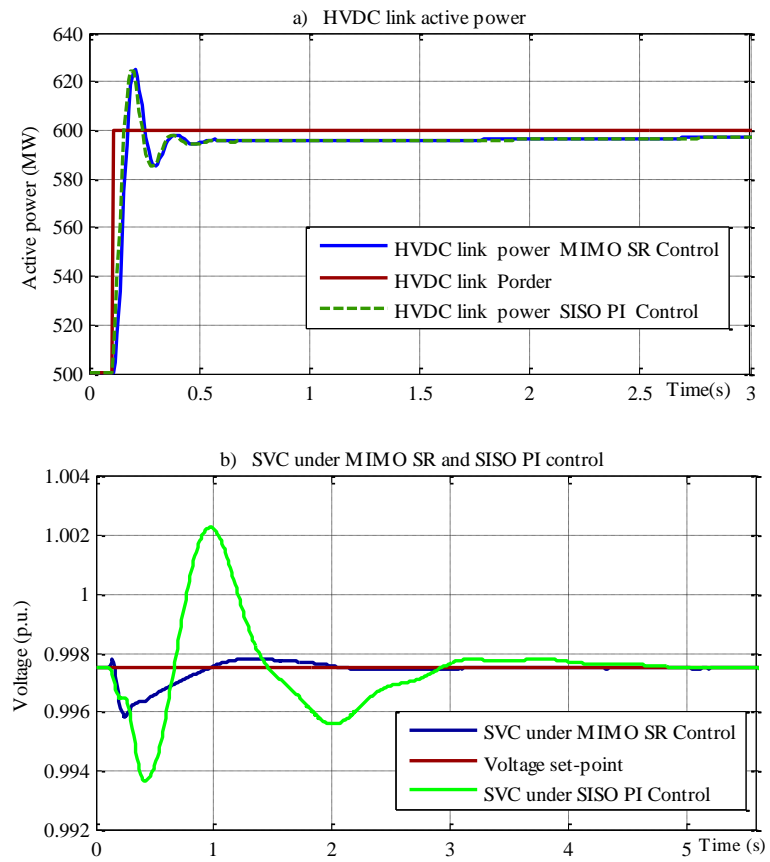

Figure 5. Comparison of SISO PI and MIMO SR control performance for coordinated control of SVC and HVDC link.

\section{CONCLUSIONS}

A MIMO Sample Regulator control method was developed. Its capability for coordinated control of power flow control devices such as HVDC link and SVC at pre-fault was demonstrated using two power system applications, including coordination of power flow control of HVDC links and power flow control of HVDC with the SVC voltage regulation. Also, robustness of this class of sampled regulator has been tested at post-fault which confirmed that the sampled regulators design method is both practical and applicable to power system control even when the systems are subjected to severe disturbances. Therefore, by adopting a MIMO SR controller, the HVDC link can also provide rapid post fault action to pick up the flow on the lost $\mathrm{AC}$ line and consequently restore system stability.

\section{FUTURE WORK}

Since the Anglo-Scottish boundary is such a critical interconnection, both traditionally (as it is limited by stability constraints) and forthcoming (due to the increasing renewable generation growth), NG have put a huge investment such as commissioning an embedded HVDC link and TCSC into this area to upgrade the network to increase power transfer capability across this boundary in order to accommodate the future renewable generation. As a result, an overall closed loop stability control system is required that firstly could reconcile with existing tools and control systems and secondly cater for the need of all upcoming and existing power flow and voltage control devices such as SVC, HVDC link with their associated POD control . Also, such a system would ideally be aimed to address the issues of stability, coordination and optimization concurrently. The HVDC link and SVC are both fast and automatic controlled devices with different control objectives. On the whole the SVC regulates the voltage and HVDC link primarily controls pre-fault power flow while at the immediate post fault the HVDC link fast ramping up dynamic and short term over- load capabilities can be utilized to enhance transient stability across Scottish boundary. The next step of this research is to define the overall control strategies for such a stability system under a single frame work. In addition to developing the closed loop MIMO Sample Regulator to provide coordinated control of HVDC links, SVC and TCSC under one framework at pre-fault and enhance the boundary stability limit at the post fault using HVDC link post fault action. Finally, further to theoretical development, the control designed method will be tested on the full GB transmission system for coordinated control of various types of control devices.

\section{ACKNOWLEDGMENT}

The authors gratefully acknowledge the funding provided to this Doctorate scheme by the Engineering and Physical Sciences Research Council (EPSRC) and National Grid, UK.

\section{REFERENCES}

[1] Commission of the European Communities "2020 by 2020 Europe's Climate Change Opportunity". [Online]. Available: http://www.energy.eu/directives.

[2] "The Climate Change Act". [Online]. Available: http://www.legislation.gov.uk/ukpga/2008.

[3] ENSG, "Our Electricity Transmission Network: A Vision for 2020" [Online]. Available: http://webarchive.nationalarchives.gov.uk/ 2009.

[4] Åström, K. J. "A robust Sampled Regulator for Stable Systems with Monotone Step Responses," Automatica, 16, pp313, 1980.

[5] Mossaheb, S., Cameron, R. G. and Li, F." Passivity and the Design of Sampled Regulators for Unknown Systems," Int. J. Control, 45, pp1941, 1987.

[6] Wang, H. F., Li, F. and Cameron, R. G. "FACTS Control Design Based on Power System Nonparametric Models," IEE 19990545, 1999.

[7] Wang, H. F. and Li, F., "Multivariable sampled regulators for the coordinated control of STATCOM AC and DC voltage," IEE Proc.Gener., Transm and Distrib. Vol147-2 pp93, 2000.

[8] Kerahroudi, S.K.; F.Li, Taylor, G.A.; Bradley, M ."Initial Development of a Novel Operational Stability Control system in the Future GB Transmission System," Universities Power Engineering Conf (UPEC), 2013 48th International, pp.1, 6, 4-7 Sept. 2013.

[9] PowerFactory DIgSILENT manual.

[10] Kerahroudi, S.K.; Zobaa, A.F.; Taylor, G.A.; Bradley, "Framework of the Coordinated Stability Control in the Future GB Transmission System Using HVDC and Power Flow Controller Devices" IET ACDC Conf, November 2012. 